\title{
CARACTERIZACIÓN DE LA FUNCIONALIDAD FAMILIAR Y REDES SOCIALES EXISTENTES EN DESPLAZADOS POR LA VIOLENCIA EN ALGUNAS LOCALIDADES DE BOGOTÁ DC.
}

\section{AÑO 2007, BAJO LOS LINEAMIENTOS DE MEDICINA FAMILIAR}

Ivonne Andrea Rincón Rueda MD*, Amarilis María Cantillo Meléndez MD*, José Fernando Ordúz Sánchez MD $^{* *}$, Silvia Lorena Mora Villa-Roel MD***

\section{Resumen}

Introducción: Bogotá DC. como capital de Colombia es el receptor del mayor flujo migratorio de población desplazada por causa de la violencia, son individuos o grupos familiares con crisis no esperadas, que requieren una atención integral para restituir su bienestar biopsicosocial. Objetivo: caracterizar la funcionalidad, ciclo vital familiar e identificar las redes sociales existentes, por medio de instrumentos de medicina familiar, de una población desplazada por la violencia, en algunas localidades de Bogotá DC. durante el año 2007. Métodos: con la información de las carpetas familiares del convenio de la Fundación Universitaria de Ciencias de la Salud (FUCS) y ASCOFAME, se realizó un subanálisis de la información en un estudio descriptivo de corte transversal, en el cual se describen las características demográficas y la funcionalidad familiar medida por instrumentos debidamente validados (APGAR familiar), así como la valoración de las redes sociales existentes en familias desplazadas por la violencia, certificadas por el gobierno de Colombia, en tres localidades de Bogotá DC.: Engativá, Mártires y San Cristóbal. Resultados: se describen las características de 795 familias con 3.815 individuos. La edad promedio fue 22 años (de 17), la mayor proporción de la población proviene de la zona Andina (49,9\%) y Caribe (18,9\%). El 53,5\% de las familias tenían hijos adolescentes y escolares. Con el puntaje de APGAR se encontraron 40,7\% familias funcionales, $27 \%$ con disfunción familiar leve, $20,8 \%$ moderada y $11,5 \%$ con disfunción severa. Conclusiones: la mayoría de las familias desplazadas mostraron por medio del APGAR familiar aplicado a un miembro, ser disfuncionales en cualquier grado predominando la disfunción leve. La severa se asocia con desempleo y ausencia de recreación en el núcleo familiar.

Palabras clave: desplazamiento interno en Colombia, funcionalidad familiar, APGAR familiar, familiograma, redes sociales.

\section{Abstract}

Introduction: As the capital city of Colombia, Bogotá DC. is the major receptor of population migration resulting from violence. These are individuals or family groups who experience non expected crises and need comprehensive assistance in order to recover their biological/psychosocial well-being. Objective: to characterize family function and vital cycle and to identify

Fecha recibido: marzo 8 de 2010 - Fecha aceptado:

* Residente de Medicina Familiar III año, Fundación Universitaria de Ciencias de la Salud, Bogotá DC. Colombia.

** Magíster en Salud Pública, Universidad de Antioquia. Candidato a Magíster en Medicinas Alternativas, Universidad Nacional. Director del Área de Salud Pública, Facultad de Medicina, Fundación Universitaria de Ciencias de la Salud, Bogotá DC. Colombia.

*** Especialista en Medicina Familiar, Jefe del Departamento de Medicina Social y Comunitaria, División de Investigaciones, Fundación Universitaria de Ciencias de la Salud, Hospital de San José, Bogotá DC. Colombia. 
existing social networks by means of family medicine instruments applied on a violence-related displaced population in some locations of Bogotá DC. during year 2007. Methods: a subgroup analysis was performed using data of family files of Fundación Universitaria de Ciencias de la Salud (FUCS) and ASCOFAME Agreement by means of a cross sectional descriptive trial. It describes demographic features and family function measured with duly validated instruments (Family APGAR), as well as, the assessment of current social networks in Colombian-government-certified violence-related displaced families in three locations: Engativá, Mártires and San Cristóbal. Results: the characteristics of 795 families and 3815 individuals are described. Mean age was 22 years (of 17). The greatest proportion of the studied population came from the Andean zone (49.9\%) and the Caribbean (18.9\%). Adolescents and elementary-school-aged children were found in $53.5 \%$ of families. Using the Family APGAR instrument, $\mathbf{4 0 . 7 \%}$ were classified as functional families, $27 \%$ as having mild dysfunction, $20.8 \%$ moderate and $11.5 \%$ severe dysfunction. Conclusions: any degree of dysfunction was identified in most of the displaced families, predominantly mild dysfunction, when the Family APGAR instrument was completed by one of their members. Severe dysfunction is related to unemployment and to lack of recreation within family nucleus.

Key words: internal migration in Colombia, family function, family APGAR, family graph, social networks.

\section{Introducción}

El desplazamiento forzado interno en Colombia por la violencia se define como "toda persona que se ha visto forzada a migrar dentro del territorio nacional abandonando su localidad de residencia o actividades económicas habituales, porque su vida, su integridad física, su seguridad o libertad personales han sido vulneradas o se encuentran amenazadas, por ocasión cualquiera de las siguientes situaciones: conflicto armado interno, disturbios y tensiones interiores, violencia generalizada, violaciones masivas de los derechos humanos, infracciones al derecho internacional humanitario $u$ otras circunstancias emanadas de las situaciones anteriores que puedan alterar el orden público". ${ }^{1}$

Bogotá DC. como capital de Colombia es la receptora del mayor flujo migratorio de población desplazada por causa de la violencia con cerca del $23 \%$ del total de desplazados. En esencia son individuos o grupos familiares que tienden a concentrarse en las localidades más pobres. La mayoría llegan con la ilusión de iniciar nuevos proyectos de vida, desarrollar una actividad productiva y generar recursos para poder acceder a los servicios básicos, así como a una vivienda, condiciones de vida dignas y seguridad para su familia. ${ }^{2}$

El desplazamiento forzado genera en el núcleo familiar cambios evidentes en los roles de cada miembro, deserción escolar de los hijos, cambios económicos y de su idiosincrasia, entre otros; características que son llamadas crisis no normativas, eventos no esperados dentro del desarrollo de los ciclos vitales de la familia. Estas crisis desestructuran la familia, su dinámica y las redes de apoyo que se han conformado por años con individuos y familias, que cambian su proyecto de vida con la posibilidad de generar posibles enfermedades mentales y orgánicas. ${ }^{3}$

Estos efectos sobre la familia y sus miembros hacen necesario describir, diagnosticar o caracterizar esta problemática bajo un enfoque familiar, que no sólo debe tener efectos sociales y estatales a favor de las familias desplazadas, sino que como ente universitario se compromete a ver la medicina desde un punto de vista comunitario y de familia, importante en el enfoque de promoción de la salud y prevención de la enfermedad como una de las maneras más apropiadas de modificar la morbimortalidad y la calidad de vida de la población.

La Asociación Colombiana de Facultades de Medicina (ASCOFAME), en convenio con la Fundación Universitaria de Ciencias de la Salud (FUCS), desarrollan un proyecto llamado Programa para el acompañamiento y tutoría a desplazados con enfoque en salud familiar, con el objeto de restituir el bienestar biopsicosocial de familias desplazadas direccionándolas a redes de apoyo que el gobierno $u$ otras entidades ofrezcan, fortaleciendo además las competencias académicas en medicina y salud familiar de los estudiantes de la universidad. Este programa culminó para ASCOFAME a finales de 2008, pero continúa haciendo parte del currículo académico del área de medicina social y comunitaria de la Facultad de Medicina.

El proyecto generó para la FUCS dos tipos de productos: la prestación de servicios de salud para desplazados como política pública y algunos procesos académicos. 
Dentro del primero están el fortalecimiento del trabajo intersectorial e interinstitucional, la oferta asistencial en salud con enfoque biopsicosocial, las redes de apoyo social, la atención domiciliaria y el apoyo familiar, además de contribuir al posicionamiento del modelo de medicina familiar como estrategia para el desarrollo de la atención primaria en población vulnerable. En el segundo es importante destacar la adecuación curricular de la universidad, el fortalecimiento del trabajo interfacultades de programas universitarios que tienden a mejorar la calidad de vida de la población, la investigación académica con proyección social y el fortalecimiento de la formación de talento humano en salud con enfoque biopsicosocial.

El objetivo del presente estudio fue caracterizar la funcionalidad, identificar el ciclo vital familiar y las redes sociales existentes, por medio de instrumentos de medicina familiar, en una población desplazada por la violencia en algunas localidades de Bogotá, durante el año 2007.

\section{Materiales y métodos}

En el desarrollo de dicho programa se realizó un estudio descriptivo de corte transversal con familias desplazadas por la violencia captadas en tres localidades de Bogotá: Engativá, Mártires y San Cristóbal, a las que se les realizó una encuesta registrada en carpetas familiares, entre enero y diciembre de 2007. A partir de estos datos se realizó un subanálisis de la información, incluyendo sólo familias desplazadas certificadas por acción social del gobierno colombiano, independiente del tiempo de desplazamiento y conformadas por dos o más miembros. Las encuestas fueron realizadas previo entrenamiento del grupo interdisciplinario (estudiantes de las facultades de medicina y enfermería, residentes e internos de medicina familiar y el Departamento de Psicología) explorando las características demográficas y la funcionalidad familiar medida por un instrumento previamente validado (APGAR familiar) ${ }^{4}$ así como la valoración de las redes sociales de apoyo existentes en las familias.

Los objetivos se clasificaron de acuerdo con el análisis de individuos y familias. La descripción de los primeros se centra en las características demográficas: edad, ci- clo vital, sexo, nivel de escolaridad y tipo de afiliación al sistema de seguridad social en salud (contributivo, subsidiado o vinculado). Para la descripción de familias se tuvo en cuenta el lugar de procedencia definido como el departamento de origen, clasificado según las regiones geográficas de Colombia (Andina, Caribe, Pacífica, Amazonia y Orinoquia), número de integrantes por familia, ciclo vital familiar, ${ }^{3}$ tenencia de vivienda (propia, alquilada, invasión u otra), acceso a servicios públicos (disponibilidad de agua, luz y alcantarillado), familia extensa (autorreporte de la presencia de familia no nuclear que apoya de cualquier forma), religión (autorreporte de cualquier creencia religiosa), recreación (autopercepción de acceso a espacios y tiempo para esparcimiento y distracción compartidos por la familia al momento de la encuesta), servicios de salud (acceso de todos los miembros de la familia a servicio de salud), educación (posibilidad de educación de todos los niños y adolescentes miembros de la familia), seguridad alimentaria (contar mínimo con tres comidas diarias para todos los miembros de la familia) y trabajo (cuando algún miembro trabaja y permite un ingreso mensual, formal ó informal).

El APGAR familiar se evaluó en un miembro de cada familia (independiente de su condición de cabeza de hogar), explorando dominios de comunicación, afecto, apoyo, participación y tiempo libre; puntúa entre 0 y 20 , correspondiendo las cifras bajas a disfunción severa y las altas a familia funcional. ${ }^{4}$ Se exploró la asociación entre APGAR y la presencia de redes sociales de apoyo.

La base de datos se realizó en Excel 2003 y el análisis estadístico en STATA 10®. Se presenta un análisis descriptivo reportando frecuencias absolutas y porcentajes para variables categóricas y se resumen los datos cuantitativos mediante promedio y desviación estándar. La exploración de asociación entre variables se expresa mediante análisis de correspondencias múltiples con el programa $\mathrm{R}$, con el cual se exploró en forma descriptiva la asociación entre funcionalidad y las redes de apoyo social. Se encontró asociación entre funcionalidad familiar y acceso a educación, recreación, trabajo, familias con adolescentes y escolares. La disfunción familiar severa se encuentra asociada con falta de recreación o trabajo, familias en formación y con preescolares. Se utilizó la prueba ji-cuadrado para confirmar dicha aso- 
ciación. El estudio fue aprobado por el comité de investigaciones y ética de la Fundación Universitaria de Ciencias de la Salud.

\section{Resultados}

Se describen las características de 795 familias representadas por 3.815 individuos captados durante la ejecución del proyecto (Figura 1). La Tabla 1 presenta la información de las familias. De acuerdo con la región de procedencia la mayor proporción corresponde a población en condición de desplazamiento de la zona Andina, (49,9\%) seguida de la Caribe (18,9\%) y la Pacífica (12,2\%). El número de integrantes por familia en promedio fue cuatro (DE:1.7), el 50,6\% tenían entre cinco y nueve miembros. El 30,7\% de las familias tenían hijos adolescentes y el $22,8 \%$ escolares, mientras que las familias en etapa de formación de la pareja sólo representaban el $1,7 \%$ de la población estudiada.

La vivienda en arriendo fue reportada por el $87,3 \%$, el $7,2 \%$ contaba con vivienda propia y el $3,2 \%$ vivía en zona de invasión. El acceso a servicios públicos básicos estaba disponible para la mayoría de familias, permaneciendo el $5 \%$ de población carente de algunos de éstos. La tenencia de trabajo formal o informal en por lo menos un miembro de la familia fue documentada en el 67,4\% de las familias. El acceso a salud fue reportado por el $88,5 \%$ de las familias, educación 78,7\% y recreación el 58,8\%. Cuando se caracterizó la población de acuerdo con el puntaje de APGAR se encontraron $40,7 \%$ de familias funcionales, $27 \%$ con disfunción familiar leve, $20,8 \%$ con disfunción familiar moderada y el 11,5\% con disfunción familiar severa.

La Tabla 2 presenta las características de los 3.815 individuos, encontrando edad promedio de 22 años (DE 17.0) con un rango muy amplio, desde el mes de nacido hasta 92 años. Fue evidente que la población está representada en su mayoría por adultos jóvenes y adolescentes, 33,2\% y $27,3 \%$ respectivamente. La distribución por sexo muestra una proporción de mujeres de $53,4 \%$. Se documentó para el momento de la encuesta el 3,9\% de la población sin ningún grado de escolaridad, aunque un
Tabla I. Características de familias

\begin{tabular}{|c|c|c|}
\hline $\begin{array}{l}\text { Procedencia por regiones } \\
\text { colombianas n (\%) }\end{array}$ & $\mathbf{n}$ & (\%) \\
\hline Zona Andina & $392 / 785$ & $(49,9)$ \\
\hline Zona Caribe & $148 / 785$ & $(18,9)$ \\
\hline Zona Amazonia & $83 / 785$ & $(10,6)$ \\
\hline Zona Orinoquia & $66 / 785$ & $(8,4)$ \\
\hline Zona Pacífica & $96 / 785$ & $(12,2)$ \\
\hline \multicolumn{3}{|l|}{ Ciclo vital familiar } \\
\hline Formación de la pareja & $13 / 765$ & $(1,7)$ \\
\hline Crianza inicial & $52 / 765$ & $(6,8)$ \\
\hline Con hijos preescolares & $81 / 765$ & $(10,5)$ \\
\hline Con hijos escolares & $175 / 765$ & $(22,8)$ \\
\hline Con hijos adolescentes & $235 / 765$ & $(30,7)$ \\
\hline En plataforma de lanzamiento & $159 / 765$ & $(20,7)$ \\
\hline En edad mediana* & $18 / 765$ & $(2,3)$ \\
\hline Anciana & $32 / 765$ & $(4,1)$ \\
\hline \multicolumn{3}{|l|}{ Tenencia de vivienda } \\
\hline Propia & $56 / 778$ & $(7,2)$ \\
\hline Alquilada & $679 / 778$ & $(87,3)$ \\
\hline Invasión & $25 / 778$ & $(3,2)$ \\
\hline Otros & $18 / 778$ & $(2,3)$ \\
\hline \multicolumn{3}{|l|}{ Tenencia de servicios públicos } \\
\hline Tiene luz, agua y alcantarillado & $739 / 778$ & $(95,0)$ \\
\hline $\begin{array}{l}\text { No tiene alguno de los servicios } \\
\text { mencionados }\end{array}$ & $39 / 778$ & $(5,0)$ \\
\hline \multicolumn{3}{|l|}{ Familia extensa } \\
\hline Tiene apoyo familiart & $389 / 616$ & $(63, I)$ \\
\hline \multicolumn{3}{|l|}{ Religión } \\
\hline Católica & $518 / 736$ & $(70,4)$ \\
\hline Cristiana & $123 / 736$ & $(16,7)$ \\
\hline Otras $\ddagger$ & $37 / 736$ & $(5,0)$ \\
\hline No tiene & $58 / 736$ & $(7,9)$ \\
\hline \multicolumn{3}{|l|}{ Recreación } \\
\hline Tiene $\S$ & $428 / 728$ & $(58,8)$ \\
\hline \multicolumn{3}{|l|}{ Servicios de salud\| } \\
\hline Tiene & $681 / 769$ & $(88,5)$ \\
\hline \multicolumn{3}{|l|}{ Educación ף } \\
\hline Tiene & $581 / 738$ & $(78,7)$ \\
\hline \multicolumn{3}{|l|}{ Seguridad alimentaria $* *$} \\
\hline Tiene & $567 / 736$ & $(77,0)$ \\
\hline No tiene & $169 / 736$ & $(22,9)$ \\
\hline \multicolumn{3}{|l|}{ Trabajo $+\dagger$} \\
\hline Tiene & $517 / 767$ & $(67,4)$ \\
\hline \multicolumn{3}{|l|}{ APGAR familiar } \\
\hline Disfunción severa (0-9) & $85 / 735$ & $(\mid 1,5)$ \\
\hline Disfunción moderada (I0-13) & $153 / 735$ & $(20,8)$ \\
\hline Disfunción leve (14-17) & $198 / 735$ & $(27,0)$ \\
\hline Funcional $(18-20)$ & $299 / 735$ & $(40,7)$ \\
\hline \multicolumn{3}{|c|}{ Número de integrantes de la familia } \\
\hline 4 ó menos & $386 / 795$ & $(48,5)$ \\
\hline 5 a 9 & $402 / 795$ & $(50,6)$ \\
\hline 10 más & 7/795 & $(0,9)$ \\
\hline
\end{tabular}

* Corresponde a familia posparental; $\dagger$ si cuenta con apoyo fuera del núcleo familiar; $\ddagger$ que pertenece a otra religión y no aclara cuál; $\S$ cualquier momento de esparcimiento en familia; \| cualquier forma de atención en salud; $\mathbb{T}$ que los niños, adolescentes y adultos jóvenes que en el momento estén recibiendo cualquier tipo de educación; ** el núcleo familiar cuenta con tres comidas diarias mínimo; †† formal y/o informal de cualquier miembro de la familia. 


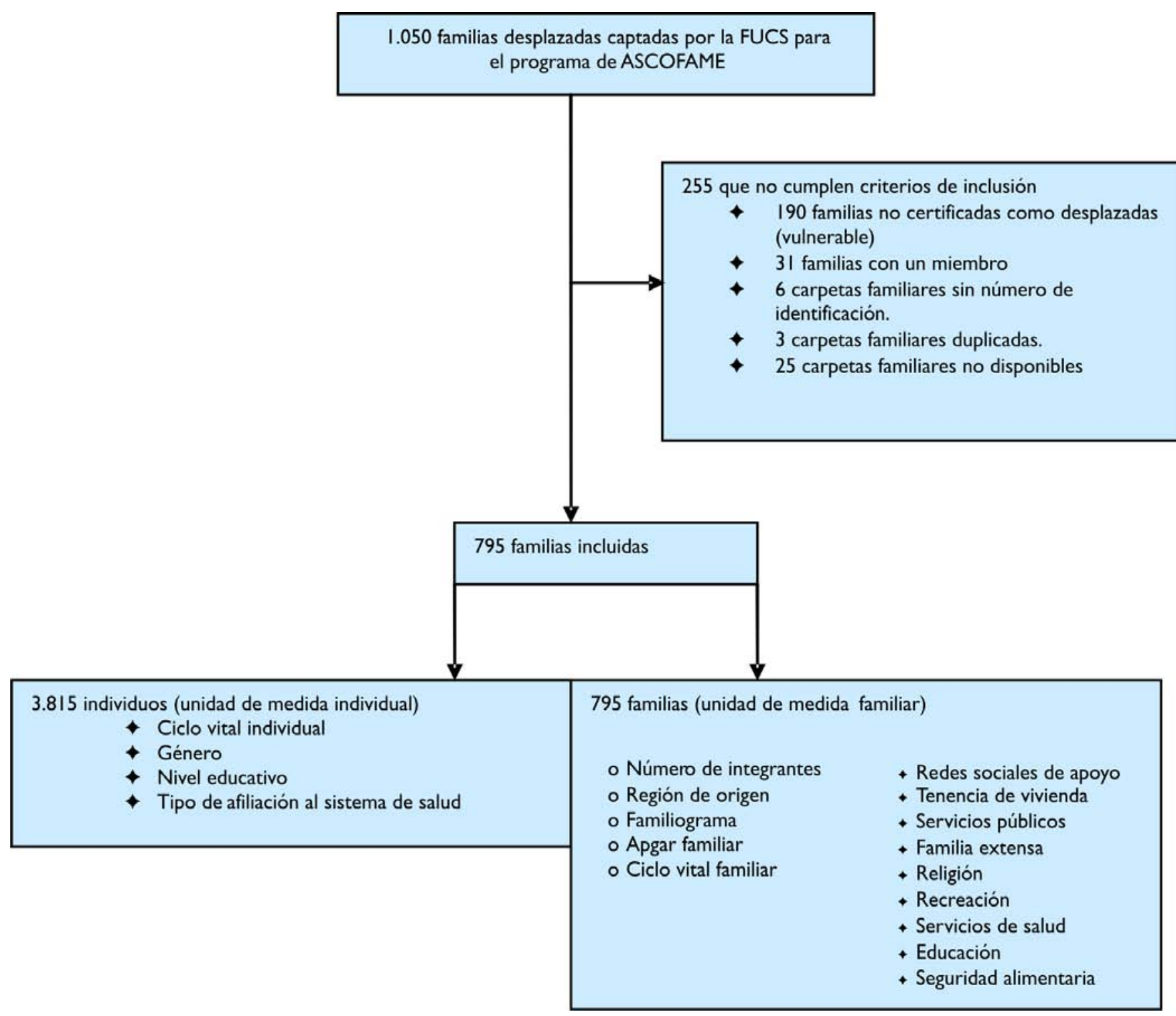

Figura I. Características de las familias.

$30,7 \%$ contaba con secundaria incompleta. Sólo un 2,1\% alcanzó estudios técnicos completos. La mayor proporción de los individuos se encontraba registrado en condición de vinculado al sistema de seguridad social en salud correspondiendo a un $56,5 \%$ de la población y sólo un $4,7 \%$ estaba en el régimen contributivo. Se exploró la relación entre la funcionalidad, medida por APGAR familiar y las redes de apoyo sociales, encontrando asociación estadísticamente significativa con trabajo y recreación (Tabla 3).

\section{Discusión}

Los principales resultados de esta investigación se dan en la relación de la funcionalidad familiar con la tenencia de trabajo y recreación, características que resultan importantes dentro de la dinámica familiar porque son fuente del sustento diario para vivir y permiten generar un proyecto de vida, salud mental, integración familiar, adaptación social y participación comunitaria, disminuyendo así el desempleo global e inseguridad de una ciudad. ${ }^{5,6,7}$ No se encontraron referencias que re- 
Tabla 2. Características de los individuos

Edad (años promedio y DE*)
Rango mínimo (meses) - máximo (años)

Ciclo vital individual, $\mathbf{n}(\%)$

Lactante 0-2 años

Preescolar 3-5 años

Escolar 6-9 años

Adolescente 10-20 años

Adulto joven $21-44$ años

Adulto maduro 45-59 años

Adulto mayor $=60$ años

Sexo, $\mathbf{n}(\%)$

Mujeres

Hombres

Nivel educativo, $\mathbf{n}(\%)$

Ninguno

Primaria incompleta

Primaria completa

Secundaria incompleta

Secundaria completa

Técnico incompleto

Técnico completo

Tipo de afiliación a salud $\dagger, \mathbf{n}(\%)$

Contributivo

Subsidiado

Vinculado

* Desviación estándar; † contributivo si aporta al sistema de salud como trabajador, subsidiado si el sistema subsidia la salud o vinculado si el individuo es registrado como desplazado, tiene derecho a acceso a salud.

Tabla 3. Factores asociados con funcionalidad dada por APGAR familiar

\begin{tabular}{|l|c|}
\hline Factor asociado & P valor \\
\hline Trabajo & 0.003 \\
\hline Recreación & 0.004 \\
\hline
\end{tabular}

porten la asociación entre recreación y funcionalidad familiar; se considera importante tenerla en cuenta en futuros estudios como elemento significativo en la función familiar. Por tanto, es indispensable fortalecer las diversas formas de recreación familiar y así promover una cultura en este sentido.

Con respecto al análisis del desempleo, la publicación de Castillo ${ }^{8}$ toma datos del sistema de información sobre población desplazada por la violencia en Colombia (RUT) del 2005 reportando $42,6 \%$ de desempleo y en la publicada por la serie Salud y Desplazamiento en Colombia en 2002 y 2003, realizada en seis ciudades colombianas por la Universidad de Antioquia ${ }^{9}$ refiere 52,2\%, en contraste con lo encontrado en este informe que es de $32,6 \%$. Esto podría interpretarse por el año de realización y el tamaño de la población estudiada, teniendo en cuenta que estos datos no son extrapolables a todos los desplazados del país.

La distribución por sexo en los estudios de Castillo $^{8} \mathrm{y}$ Arias y col. ${ }^{9}$ muestran proporciones similares de hombres y mujeres desplazados ( $50 \%$ y 54,9\%), lo cual corresponde con nuestras cifras (53\%). Se encontró que la mayoría de la población desplazada pertenece al grupo de adulto joven del ciclo vital individual con un $33,2 \%$, comparado con el estudio de Castillo ${ }^{8}$ que reporta un $30,8 \%$. Se cree que este grupo poblacional lo conforman familias jóvenes con hijos menores que ven mejores oportunidades para su descendencia. Con respecto al nivel educativo y departamento de origen mostró similares resultados con primaria completa y zona andina como las variables más frecuentes. Jacobsen y col. ${ }^{10}$ en población desplazada de Santa Marta (Colombia) en el 2008, presenta resultados con respecto al número de integrantes por familia acordes con la presente publicación (5.3 y 5 a 9 integrantes) lo cual indica que son familias numerosas las que tienden a desplazarse.

Con respecto a la afiliación a servicios de salud el estudio realizado en 1993 por Díez G. ${ }^{11}$ una población desplazada a Medellín muestra que sólo un $23 \%$ estaba, afiliada en su mayoría como cotizantes, mientras que en la población descrita sólo un $4,7 \%$ pertenecía al régimen contributivo y la mayoría estaban vinculados al sistema de salud sólo por su condición de desplazamiento. En tanto que el estudio de Londoño B. ${ }^{12}$ en la Universidad 
del Rosario (2004) muestra una cifra diferente en Bogotá, donde el derecho a la salud está dado a través de la carta de certificación de desplazamiento sólo para el servicio de urgencias, como situación transitoria una vez se afilien de manera formal a una EPS del régimen subsidiado o contributivo, condición que semeja al presente trabajo. La variable seguridad alimentaria fue definida en otros estudios en forma diferente. Sin embargo, en el estudio realizado por Prada y col. ${ }^{13}$ demuestra que a pesar que las familias desplazadas tienen acceso a alimentos, son de calidad insuficiente.

En Colombia se han realizado varios estudios en los cuales se emplean algunas variables para explicar características demográficas y de migración en población desplazada. Sin embargo, no existe uno que relacione la crisis no normativa de un núcleo familiar (desplazamiento forzado) con su funcionalidad en un lugar de asentamiento. Bellon J. y col. ${ }^{14}$ en 2007 publica en Family Practice un estudio de cohorte donde concluye que la funcionalidad familiar medida con APGAR es un factor predictor determinante de riesgo psicosocial aplicable e importante en cuidado primario. De la Revilla y col. ${ }^{5}$ muestra en su estudio publicado en 2007 que las redes sociales como familia extensa, ingresos económicos y amistades como red de apoyo, se asocian con el bienestar o no de la familia y el individuo, publicaciones que apoyan los resultados arrojados en el presente estudio con respecto a la funcionalidad familiar aunque no se realizaron en familias en condición de desplazamiento o migración.

El presente estudio es la línea de base para futuras investigaciones que permitan estudiar la funcionalidad familiar en momento de crisis, detectar redes de apoyo y demostrar el impacto que éstas tengan a mediano y largo plazo. Además, tiene un importante tamaño de población que permite conocer condiciones y necesidades de estas familias.

Una de las limitaciones de este estudio es que no se tomó en cuenta el tiempo de llegada de las familias que puede variar de unos días a varios años, lo que hace que cambie el acceso a diferentes redes sociales de apoyo, el ciclo vital familiar y su adaptación. Además, cabe aclarar que la recolección de los datos en el año 2007 puede variar con respecto a la situación actual de desplazamiento, no se tiene en cuenta la evolución de estas familias en la ciudad o cambios con respecto al regreso a su lugar de origen, otras crisis familiares y cambios en políticas estatales o gubernamentales, características que hacen que se puedan recomendar estudios que a partir del presente se puedan medir estas variables en estas mismas familias o inclusive otros estudios tipo cohorte donde se haga seguimiento de la familia en el tiempo para comparar su evolución. Los análisis cualitativos podrían ser útiles también para evaluar de una forma más profunda la funcionalidad familiar. Sugerimos el uso de otros instrumentos que la definan como el familiograma, ciclo de función familiar y circulo familiar ${ }^{15}$ entre otros.

Recomendamos la atención médica con conceptos y competencias culturalmente apropiados (salud-enfermedad) y ser respetuosos, reflexibles y sensibles con las poblaciones migratorias ${ }^{7}$ fortaleciendo las competencias académicas en medicina y salud familiar de los estudiantes y residentes de la universidad (FUCS).

Conclusiones: la mayoría de las familias desplazadas estudiadas mostraron por medio del APGAR familiar aplicado a un miembro, ser disfuncionales en cualquier grado predominando el leve. La disfunción severa se asocia con desempleo y ausencia de recreación en el núcleo familiar. Además, llama la atención que esta población aunque tiene derecho ante la ley a servicios como salud y educación, se encuentra que no todos tienen acceso.

Agradecimientos: a la secretaria del Departamento de Medicina Social y Comunitaria, Leidy Pinzón por su colaboración permanente en la realización del estudio y a ASCOFAME que asignó a este Departamento de la FUCS el proyecto de reclutamiento de familias en condición de desplazamiento.

\section{Referencias}

1. Forero E. El desplazamiento interno forzado en Colombia. Washington D.C: Kellogg Institute; Woodrow Wilson; Ideas para La Paz; 2003.

2. Bogotá. Alcaldía Mayor. Desplazamiento en Bogotá, una realidad que clama atención. Bogotá: La Alcaldia; 2004

3. Adolfo R. Medicina familiar y la práctica ambulatoria. 2 a ed. Buenos Aires: Médica Panamericana; 2006

4. Arias L, Alarcón M. Medicina familiar en la práctica. Cali : Centro Editorial de Catorce; 2000 
5. De La Revilla L, De Los Ríos A, Luna del Castillo J. Factores que intervienen en la producción de los problemas psicosociales. Aten Primaria. 2007; 39(6): 305-11.

6. Lozano O, Silva A. Impacto del desplazamiento forzado en el crecimiento demográfico y el desempleo en Bogotá. Revista Facultad de Ciencias Económicas: Investigación y Reflexión. 2005; 13:90-112.

7. Fuertes C, Martín MA. El inmigrante en la consulta de atención primaria An. Sist. Sanit. Navar. 2006; 29 (Supl. 1): 9-25.

8. Castillo O. Poblaciones en situación de desplazamiento en Colombia. Una revisión de las cifras del sistema de información "RUT". Cuad. Desarro. Rural. 2005; 55:29-50.

9. Arias S, Gaviria M. Comparación de la situación en salud, entre población de situación de desplazamiento y receptora, en seis ciudades. 2002-2003. Medellín: OPS, Universidad de Antioquia; 2005. Serie salud y desplazamiento en Colombia (módulo 1)

10. Jacobsen K, Howe K. Desplazamiento interno hacia áreas urbanas: estudio de caracterización realizado por Tufts - IDMC. Caso 3: Santa Martha, Colombia. Ginebra: FIC, Tufts University;2008
11. Diez G. Condiciones de salud de familias desplazadas por violencia en Urabá Revista CES Medicina. 1997; 11:1-25.

12. Londoño B. Bogotá: una ciudad receptora de migrantes y desplazados con graves carencias en materia de recursos e institucionalidad para garantizarles sus derechos. Estud. Socio-Jurídicos. 2004; 6:353-75.

13. Prada G, Herran O, Ortiz R. Patrón alimentario y acceso a los alimentos en familias desplazadas en el municipio de Girón, Santander, Colombia. Rev Panam Salud Pública. 2008; 23:257-63.

14. Bellón JA, Delgado-Sánchez A, de Dios Luna J y Lardelli-Claret P. Patient psychosocial factors and primary care consultation: a cohort study. Fam Pract. 2007; 24:562-69.

15. Yurss I. Atención a la familia: otra forma de enfocar los problemas de salud en atención primaria. Instrumentos de abordaje familiar. An. sist. sanit. Navar. 2001; 24 (supl. 2):73-82. 